\title{
A meta-analysis of randomized controlled trials comparing early and late concurrent thoracic radiotherapy with etoposide and cisplatin/carboplatin chemotherapy for limited-disease small-cell lung cancer
}

\author{
HONGYANG LU* ${ }^{*}$, LUO FANG ${ }^{*}$, XIAOJIA WANG, JUFEN CAI and WEIMIN MAO \\ Zhejiang Key Laboratory of Diagnosis and Treatment Technology on Thoracic Oncology (Lung and Esophagus), \\ Zhejiang Cancer Hospital, Hangzhou, Zhejiang 310022, P.R. China
}

Received September 30, 2013; Accepted October 17, 2013

DOI: $10.3892 / \mathrm{mco} .2014 .311$

\begin{abstract}
The aim of the present study was to determine the optimal time for concurrent thoracic radiotherapy (TRT) with etoposide and cisplatin/carboplatin (EP/EC) chemotherapy for the treatment of limited-disease small-cell lung cancer (LD SCLC). Randomized controlled trials comparing early and late concurrent TRT with EP/EC chemotherapy for the treatment of patients with LD SCLC were identified through searching databases such as MEDLINE, the Cochrane Central Register of Controlled Trials and Embase. Early thoracic radiotherapy (ERT) was defined as initiating irradiation within 30 days after chemotherapy initiation. A total of 3 eligible randomized controlled trials were identified. No significant differences in the objective response rate were detected between early and late concurrent TRT [risk ratio (RR) $=1.01 ; 95 \%$ confi-
\end{abstract}

Correspondence to: Professor Weimin Mao, Zhejiang Key Laboratory of Diagnosis and Treatment Technology on Thoracic Oncology (Lung and Esophagus), Zhejiang Cancer Hospital, 38 Guangji Road, Banshanqiao, Hangzhou, Zhejiang 310022, P.R. China

E-mail:weiminmao@163.com

*Contributed equally

Abbreviations: TRT, thoracic radiotherapy; EP, etoposide and cisplatin; EC, etoposide and carboplatin; LD, limited-disease; SCLC, small-cell lung cancer; ERT, early thoracic radiotherapy; VALSG, Veterans Administration Lung Study Group; ED, extensive-disease; IP, irinotecan and cisplatin; IC, irinotecan and carboplatin; MST, median survival time; AHTRT, accelerated hyperfractionated thoracic radiotherapy; CEV, cyclophosphamide, epirubicin and vincristine; ASCO, American Society of Clinical Oncology; ESMO, European Society for Medical Oncology; LRT, late thoracic radiotherapy; RR, risk ratio; $\mathrm{CI}$, confidence interval; IGRT, image-guided radiation therapy; IMRT, intensity-modulated radiation therapy; VMAT, volumetric-modulated arc therapy; 4-D, four-dimensional

Key words: small-cell lung cancer, timing radiotherapy, limited stage, meta-analysis dence interval $(\mathrm{CI})$ : 0.86-1.18; $\mathrm{P}=0.90]$. Similar results were observed in the 1-, 2-, 3- and 5-year survival rates between early and late concurrent TRT $(\mathrm{RR}=1.06,95 \% \mathrm{CI}$ : 0.88-1.27, $\mathrm{P}=0.56 ; \mathrm{RR}=1.15,95 \% \mathrm{CI}: 0.77-1.71, \mathrm{P}=0.49 ; \mathrm{RR}=0.90$, 95\% CI: 0.66-1.22, $\mathrm{P}=0.49$; and $\mathrm{RR}=1.18,95 \% \mathrm{CI}: 0.64-2.16$, $\mathrm{P}=0.60$, respectively). The total incidence of grade $3-4$ adverse events, including anemia, leukopenia, neutropenia, thrombocytopenia, nausea and vomiting, infection, esophageal toxicity, pulmonary toxicity, alopecia and hemorrhage with early concurrent TRT was significantly higher compared to that with late concurrent TRT $(\mathrm{RR}=1.21,95 \% \mathrm{CI}: 1.03-1.43$, $\mathrm{P}=0.02$ ). Thus, the results of our study indicated that the prognosis of LD SCLC treated with late concurrent TRT and $\mathrm{EP} / \mathrm{EC}$ chemotherapy is similar to that with early concurrent TRT, although the incidence of grade 3-4 adverse events was lower in LD SCLC patients treated with late concurrent TRT combined with EP/EC chemotherapy.

\section{Introduction}

Lung cancer is one of the most common types of cancer and the most common cause of cancer-related mortality worldwide (1). The proportion of small-cell lung cancer (SCLC) has decreased from $17.26 \%$ in 1986 to $12.95 \%$ in 2002 (2). In 2008, 32,000 SCLC cases were diagnosed in the United States (3). SCLC is usually staged according to the Veterans Administration Lung Study Group (VALSG) staging system (4), according to which patients are classified as having limited or extensive disease (LD and ED, respectively). LD is defined as disease confined to one hemithorax, in the absence of a malignant effusion, with disease that may be encompassed in one radiation port. Disease that does not meet these criteria is defined as ED. Despite a modest improvement in survival, the outcome of SCLC remains poor. There are currently no effective targeted agents that have been approved for the treatment of SCLC (5) and chemotherapy is the cornerstone of the treatment for SCLC. In ED SCLC, the most commonly used initial combination chemotherapy regimens are etoposide combined with cisplatin (EP), etoposide combined with carboplatin (EC), irinotecan combined with cisplatin (IP) and irinotecan combined with carboplatin (IC). 
Table I. Characteristics of the included studies.

\begin{tabular}{lccccc}
\hline & \multicolumn{5}{c}{ Median } \\
Trials (refs.) & $\begin{array}{c}\text { Patient no. } \\
\text { (excluded) }\end{array}$ & $\begin{array}{c}\text { age at E/L } \\
\text { (years) }\end{array}$ & $\begin{array}{c}\text { Chemo- } \\
\text { therapy }\end{array}$ & TRT schedule & PCI E/L \\
\hline Skarlos et al (15) & $86(5)$ & $61 / 60$ & EC & 45 Gy $(1.5 \times 2$ daily x 15) & Yes, only if CR 41.0/57.0\% \\
Park et al $(16)$ & $222(3)$ & $60 / 61$ & EP & $52.5 \mathrm{~Gy} / 25 \mathrm{fx}(2.1$, once daily) & Yes, only if CR or PR 49.5/55.6\% \\
Jeremic et al (20) & $107(4)$ & $59 / 59$ & EP & 54 Gy $(1.5 \times 2$ daily x 18) & Yes, only if CR or PR 98.0/84.0\% \\
\hline
\end{tabular}

E, early; L, late; TRT, thoracic radiotherapy; PCI, prophylactic cranial irradiation; EC, etoposide and carboplatin; CR, complete response; EP, etoposide and cisplatin; PR, partial response.

Approximately one-third of SCLC patients were diagnosed with LD, which has a median survival time (MST) of 15-20 months (6). The standard clinical practice is to combine chemotherapy and thoracic radiotherapy (TRT) when treating patients with LD SCLC. A previous meta-analysis of 11 randomized trials including patients treated with chemotherapy and TRT, demonstrated an improved 2-year survival of 5.4\% and an intrathoracic tumor control rate of $25.3 \%$ (7). EP plus accelerated hyperfractionated thoracic radiotherapy (AHTRT) followed by 3 cycles of IP failed to demonstrate a survival advantage over 4 cycles of EP plus AHTRT, which remains the standard treatment for LD SCLC. An IP regimen cannot be routinely recommended for LD SCLC (8). EP is superior to cyclophosphamide, epirubicin and vincristine (CEV) in treating LD SCLC patients (9). Previous studies on cyclophosphamide-based therapy for LD SCLC failed to demonstrate any survival benefit with the addition of TRT (10-11). Carboplatin appears to be as effective as cisplatin and the EC regimen was associated with significantly lower toxicity in patients with SCLC (12). Furthermore, a previous meta-analysis of individual patient data reported no differences in efficacy between cisplatin and carboplatin as first-line treatment for SCLC; however, the incidence of hematological toxicity was higher with carboplatin and that of non-hematological toxicity was higher with cisplatin (13). In patients with LD SCLC, the most commonly used initial combination chemotherapy regimens are EP and EC. Concurrent TRT with chemotherapy has been considered as the optimal treatment for LD SCLC (14-16). However, the optimal initiation time for TRT has not been definitively determined (16). The limitations regarding early initiation of TRT are the potentially enlarged radiation fields, due to initial planning for bulky tumors, and toxicity. The aim of this meta-analysis was to determine the optimal time for combining TRT with chemotherapy $(\mathrm{EP} / \mathrm{EC})$ for the treatment of LD SCLC patients.

\section{Patients and methods}

Research objectives. The primary objective of this study was to compare the effects of early and late concurrent TRT with EP/EC on overall survival in LD SCLC. Furthermore, we aimed to evaluate early and late TRT with chemotherapy regarding objective response and the incidence of side effects.

Search strategy. The studies were selected from the following databases: MEDLINE (1966 to present), the Cochrane Central
Register of Controlled Trials (CENTRAL, 2013, Issue 5), Embase (1974 to present) and CINAHL (1982 to present). The Cochrane Lung Cancer Groups Specialized Register was searched. The reference lists of the identified studies were also searched for any additional relevant studies. The electronic search for clinical trials was complemented by a manual search of the following oncology journals: Radiotherapy and Oncology (1985 to present); International Journal of Radiation, Oncology, Biology and Physics (1985 to present); Clinical Oncology (1999 to present); Lung Cancer (1985 to present); Journal of Clinical Oncology (1985 to present); and Thorax (1985 to present). The abstracts from the annual meetings of the American Society of Clinical Oncology (ASCO) and the European Society for Medical Oncology (ESMO) from 2000 onwards were hand-searched. Colleagues, collaborators and other experts in the field were asked to identify missing and unreported trials. The search was conducted without language restrictions.

Criteria for study selection. Studies eligible for inclusion in this meta-analysis were randomized controlled clinical trials that compared early to late concurrent TRT, fully published in journals and relevant scientific meetings, for which full details were available. The patients were required to have histologically and cytologically confirmed LD SCLC. Radiotherapy was administered concurrently with chemotherapy and the chemotherapeutic regimen was EP/EC.

Early thoracic radiotherapy (ERT) was defined as initiating irradiation within 30 days after the initiation of chemotherapy (17). Late thoracic radiotherapy (LRT) was defined as initiating irradiation after 30 days following the initiation of chemotherapy.

Data extraction. The identified randomized clinical trials were assessed to determine whether they met the inclusion criteria by three independent reviewers (Lu HY, Fang L and Wang XJ). The quality of the methods and the key outcomes were evaluated against predetermined criteria. Two reviewers (Lu HY and Fang L) independently extracted data to ensure validity. Discrepancies were resolved by consulting a third reviewer (Cai JF). The following data were collected from the manuscript:patient gender, age, performance status at the time of randomization, chemotherapy regimen, induction treatment that resulted in a complete response, date of radiotherapy initiation, presence of brain or other metastases and locoregional recurrence. 


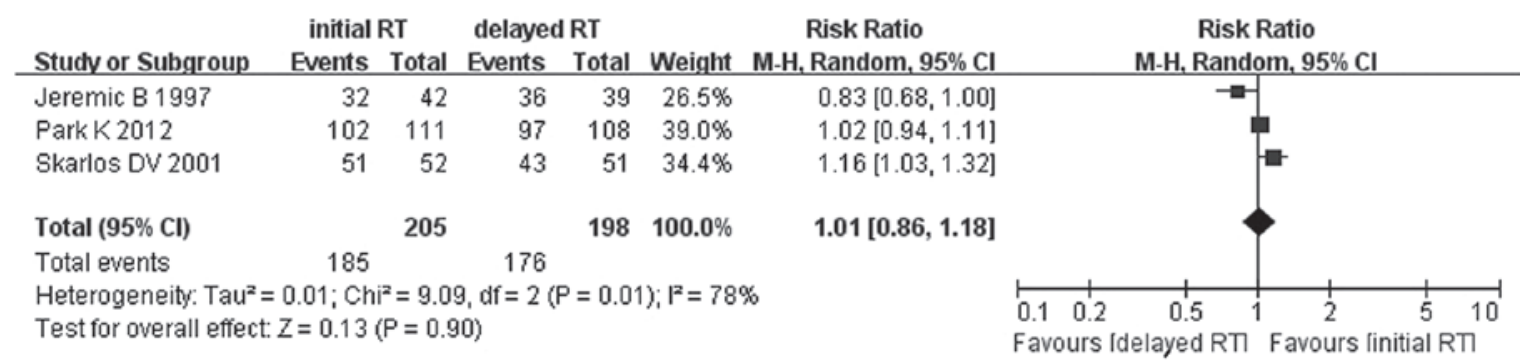

Figure 1. Forest plot of objective response comparison. There was no significant difference between the two arms [risk ratio $=1.01 ; 95 \%$ confidence interval (CI): 0.86-1.18; P=0.90]. RT, radiotherapy.

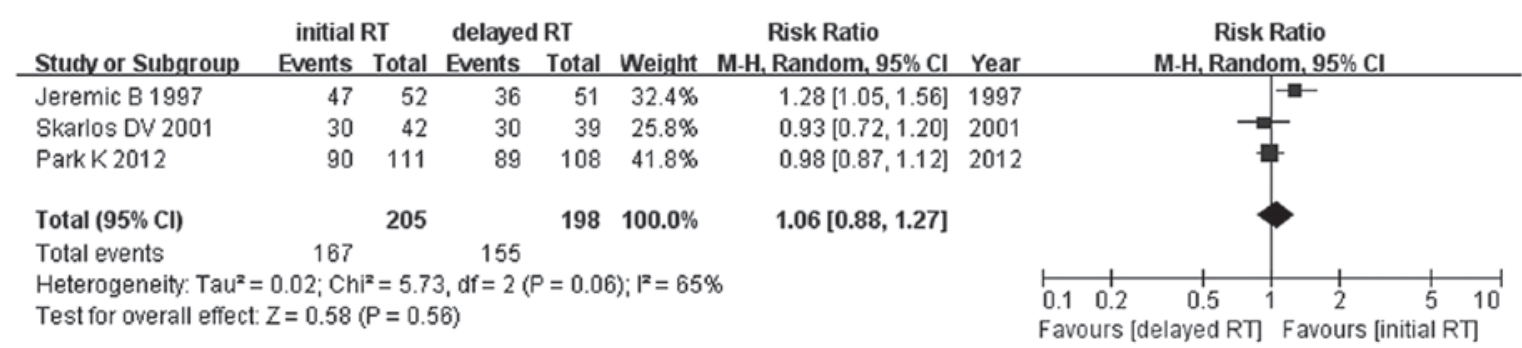

Figure 2. Forest plot of 1-year survival rates. There was no significant difference between the two arms [risk ratio = 1.06; 95\% confidence interval (CI): $0.88-1.27$; $\mathrm{P}=0.56]$. RT, radiotherapy.

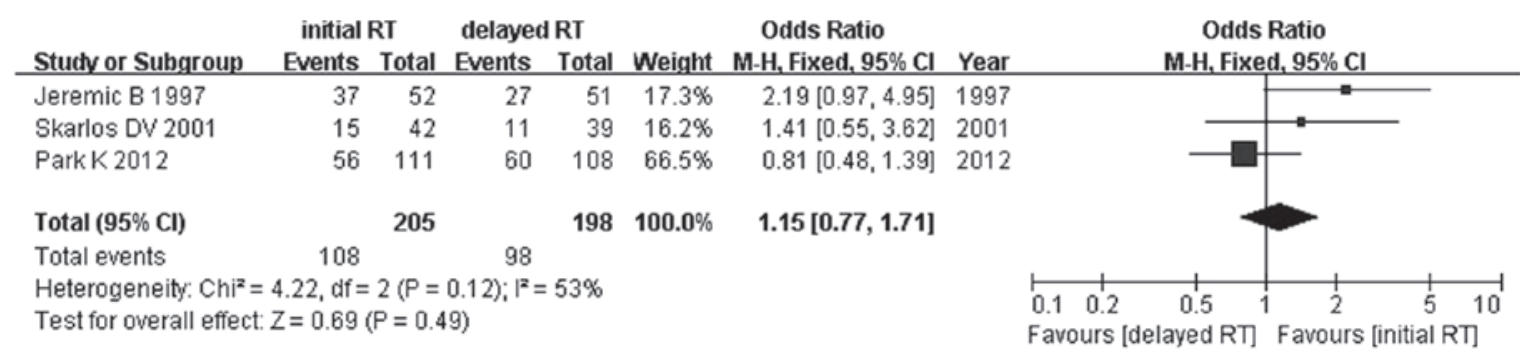

Figure 3. Forest plot of 2-year survival rates. There was no significant difference between the two arms [odds ratio $=1.15 ; 95 \%$ confidence interval (CI): $0.77-1.71$; $\mathrm{P}=0.49]$. RT, radiotherapy.

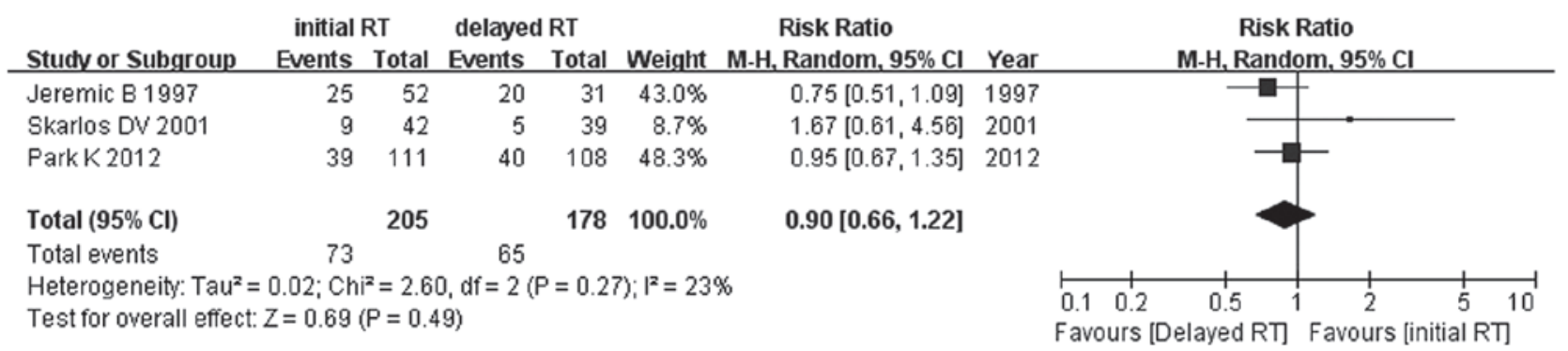

Figure 4. Forest plot of 3-year survival rates. There was no significant difference between the two arms [risk ratio $=0.90$; 95\% confidence interval (CI): $0.66-1.22$; $\mathrm{P}=0.49]$. RT, radiotherapy.

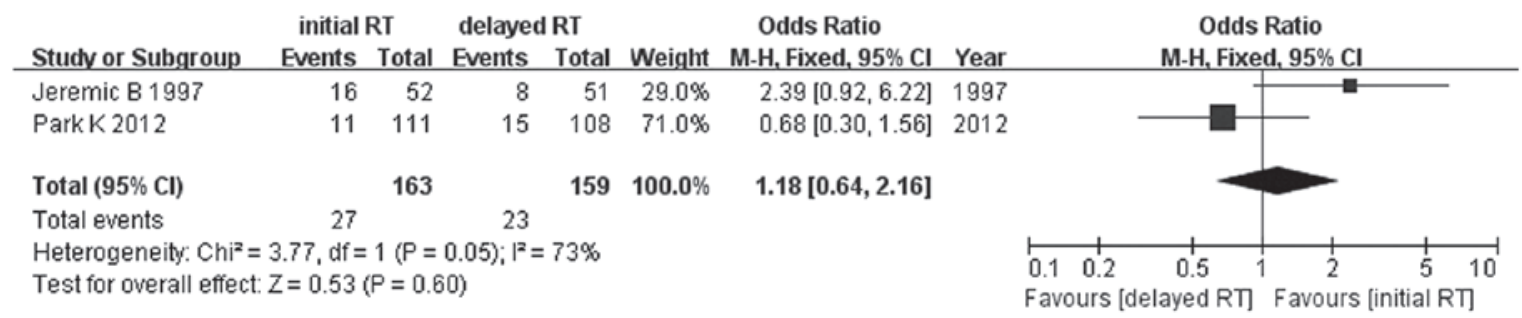

Figure 5. Forest plot of 5-year survival rates. There was no significant difference between the two arms [odds ratio = 1.18; $95 \%$ confidence interval (CI): $0.64-2.16$; $\mathrm{P}=0.60]$. $\mathrm{RT}$, radiotherapy. 


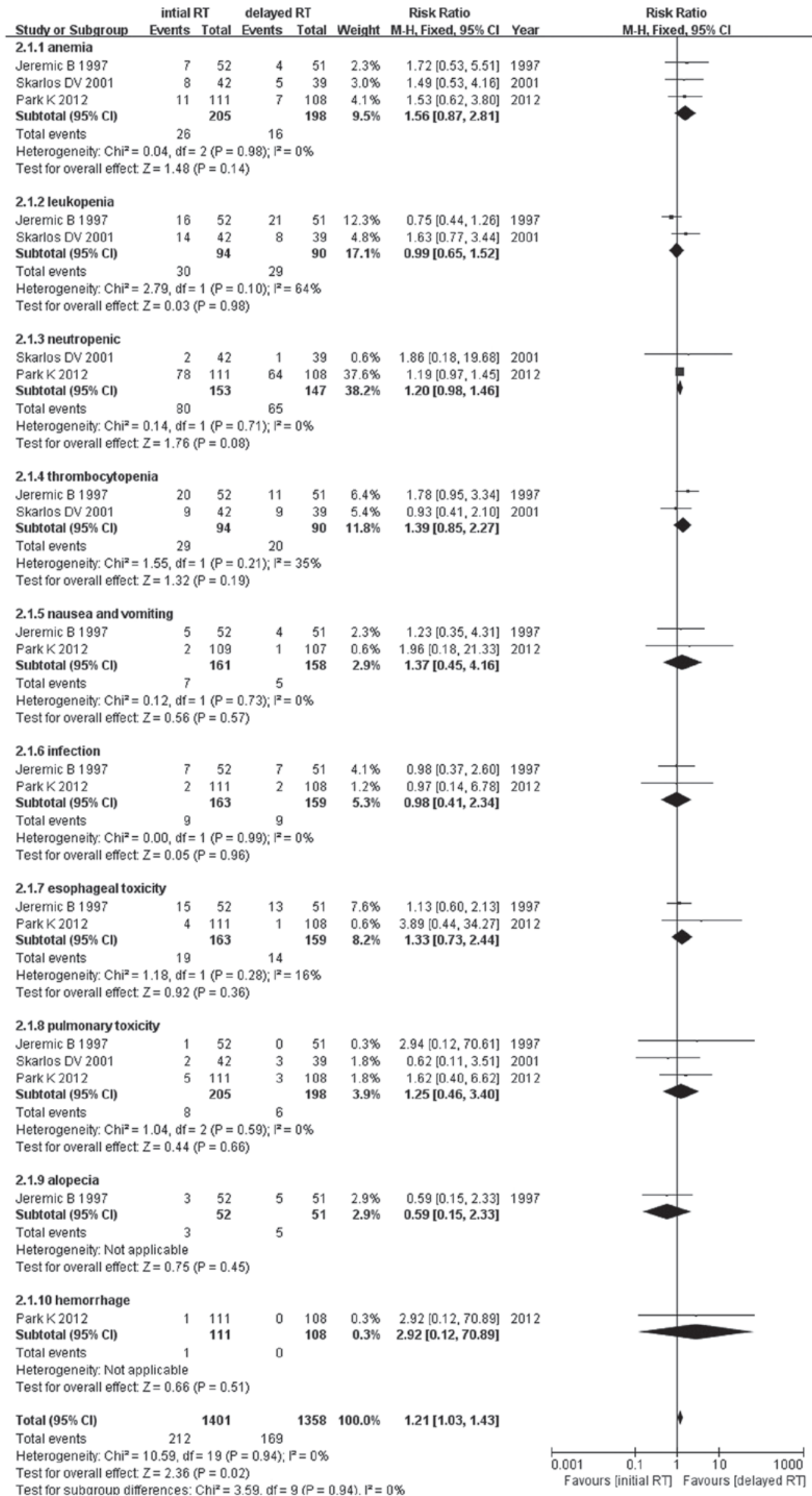

Figure 6. Forest plot of total grade 3-4 adverse events. The incidence of grade 3-4 adverse events with early thoracic radiotherapy was higher compared to that with late concurrent thoracic radiotherapy [risk ratio $=1.21 ; 95 \%$ confidence interval $(\mathrm{CI}): 1.03-1.43 ; \mathrm{P}=0.02)$. RT, radiotherapy. 
Statistical analysis. Data analysis was performed with Rev Man 5.2 software, provided by The Cochrane Collaboration. Weighted risk ratios (RRs) and their 95\% confidence intervals (CIs) were calculated according to the Mantel-Haenszel method (18). The results were assessed for heterogeneity at a significance level of $\mathrm{P}<0.05$, according to the methods of DerSimonian and Laird (19). We performed a sensitivity analysis to detect potential heterogeneity; if there was no evidence of heterogeneity, a fixed-effects model was used, whereas if heterogeneity existed, a random-effects model was used.

\section{Results}

Study selection. The characteristics of the included randomized controlled trials are summarized in Table I. The study of Park et al (16) was a phase III trial of concurrent TRT with either the first or the third cycle of EP chemotherapy in order to determine the optimal timing of TRT for LD SCLC. The study of Skarlos et al (15) was a phase II randomized comparison of early vs. late hyperfractionated TRT concurrently with EC chemotherapy in LD SCLC. The study of Jeremic et al (20) was a randomized comparison of initial vs. delayed AHTRT concurrently with EP chemotherapy for LD SCLC. Other studies were not eligible for inclusion in this meta-analysis, as the chemotherapeutic regimen was not EP/EC (21-24) and study 9104 was not considered eligible due to administration of sequential TRT in only half of the patients (14).

Comparison of early and late concurrent TRT. There were no significant differences in the objective response between early and late concurrent TRT ( RR=1.01, 95\% CI: 0.86-1.18, $\mathrm{P}=0.90$ ) (Fig. 1). Similar results were observed for 1-, 2-, 3 - and 5-year survival rates between early and late concurrent TRT $(\mathrm{RR}=1.06,95 \% \mathrm{CI}: 0.88-1.27, \mathrm{P}=0.56$; $\mathrm{RR}=1.15$, 95\% CI: $0.77-1.71, \mathrm{P}=0.49 ; \mathrm{RR}=0.90,95 \% \mathrm{CI}: 0.66-1.22$, $\mathrm{P}=0.49$; and $\mathrm{RR}=1.18,95 \% \mathrm{CI}$ : 0.64-2.16, $\mathrm{P}=0.60$, respectively) (Figs. 2-5). Since the study of Skarlos et al (15) did not provide the 5-year survival rate, only the data from Park et al (16) and Jeremic et al (20) were analyzed.

Adverse events. The incidence of grade 3-4 adverse events, such as anemia, leukopenia, neutropenia, thrombocytopenia, nausea and vomiting, infection, esophageal toxicity, pulmonary toxicity, alopecia and hemorrhage, was higher with early compared to that with late concurrent TRT $(\mathrm{RR}=1.21$, 95\% CI: 1.03-1.43, $\mathrm{P}=0.02$ ) (Fig. 6). There were no significant differences for each grade 3-4 adverse event, such as anemia, leukopenia and neutropenia (Fig. 6).

\section{Discussion}

EP and EC are the standard first-line chemotherapeutic regimens for SCLC. IP or IC may also be used as first-line chemotherapy for ED SCLC, but not for $\operatorname{LD~SCLC~}(8,25)$. The addition of TRT has improved the survival of LD SCLC patients. It was previously demonstrated that TRT combined with EP is more effective for LD SCLC compared to radiotherapy and the hematological toxicity was more severe in the concurrent arm (14). Concurrent chemoradiotherapy is preferable to sequential therapy in patients with SCLC with a good performance status. The preliminary results indicated that irradiated postchemotherapy tumor extent and omitted elective nodal irradiation did not decrease locoregional control in the study arm compared to the control arm with prechemotherapy tumor extent and the overall survival difference was not statistically significant between the two arms (26). Over two-thirds of patients who succumbed to lung cancer in the United States are aged $>65$ years (27). Elderly patients tolerate concurrent TRT poorly and toxicity must be considered for concurrent TRT in LD SCLC. It is important for LD SCLC patients to decrease the toxicity of concurrent TRT in order to complete the treatment schedule. Previous meta-analyses suggested that patients with LD SCLC may benefit from early TRT, with a significant difference if the overall treatment time of TRT is $<30$ days (17,28-30); however, the chemotherapy regimens in some clinical trials in those articles were not EP/EC. One trial demonstrated that TRT (52.5 Gy, once daily) initiated with the third cycle of chemotherapy resulted in survival outcomes and complete response rates comparable to those of TRT initiated with the first cycle of chemotherapy, with a lower frequency of febrile neutropenia (16).

In this meta-analysis, we demonstrated that there were no significant differences between early and late concurrent TRT regarding the 1-, 2-, 3- and 5-year survival rates and objective response rate, whereas the overall incidence of grade 3-4 adverse events was lower with late concurrent TRT. Elderly patients or patients with co-existing diseases should be treated with extra caution. Early concurrent TRT may result in enlarged irradiation fields, due to initial planning for bulky tumors. The balance between therapeutic effects and treatment-related toxicities should also be considered. Decreasing toxicity lowers the overall treatment cost and saves on medical resources. In order to deliver adequate radiation doses to the tumor, while respecting normal tissue dose constraints, the use of advanced technologies, including image-guided radiation therapy (IGRT), intensity-modulated radiation therapy (IMR $\mathrm{T}$ )/volumetric-modulated arc therapy (VMAT), four-dimensional (4-D) CT and/or PET-CT, is considered appropriate. Modern radiotherapy, including accurate target definition and conformal radiotherapy planning, may help maximize tumor control and minimize treatment-related toxicity.

In conclusion, we demonstrated that late concurrent TRT with EP/EC chemotherapy is suitable for LD SCLC patients, particularly elderly patients or those with bulky tumors. However, further studies on concurrent TRT with EP/EC chemotherapy for LD SCLC are required in order to increase overall survival rates and decrease treatment-related toxicity.

\section{Acknowledgements}

This study was funded by Project 81202806 supported by the National Natural Science Foundation of China and the Zhejiang Province Medical Science Fund Project of China (grant nos. 2012KYB034 and 2012RCB004).

\section{References}

1. Ferlay J, Shin HR, Bray F, et al: Estimates of worldwide burden of cancer in 2008: GLOBOCAN 2008. Int J Cancer 127: 2893-2917, 2010. 
2. Govindan R, Page N, Morgensztern D, et al: Changing epidemiology of small-cell lung cancer in the United States over the last 30 years: analysis of the surveillance, epidemiologic, and end results database. J Clin Oncol 24: 4539-4544, 2006.

3. Jemal A, Siegel R, Ward E, et al: Cancer statistics, 2008. CA Cancer J Clin 58: 71-96, 2008.

4. Patel AM, Dunn WF and Trastek VF: Staging systems of lung cancer. Mayo Clin Proc 68: 475-482, 1993.

5. Lu HY, Wang XJ and Mao WM: Targeted therapies in small cell lung cancer (Review). Oncol Lett 5: 3-11, 2013.

6. Hanna NH and Einhorn LH: Small-cell lung cancer: state of the art. Clin Lung Cancer 4: 87-94, 2002.

7. Warde $\mathrm{P}$ and Payne D: Does thoracic irradiation improve survival and local control in limited-stage small-cell carcinoma of the lung? A meta-analysis. J Clin Oncol 10: 890-895, 1992.

8. Kubota K, Hida T, Ishikura S, et al: Etoposide and cisplatin versus irinotecan and cisplatin in patients with limited-stage small-cell lung cancer treated with etoposide and cisplatin plus concurrent accelerated hyperfractionated thoracic radiotherapy (JCOG0202): a randomised phase 3 study. Lancet Oncol 15: $106-113,2014$

9. Sundstrom S, Bremnes RM, Kaasa S, et al: Cisplatin and etoposide regimen is superior to cyclophosphamide, epirubicin, and vincristine regimen in small-cell lung cancer: results from a randomized phase III trial with 5 years' follow-up. J Clin Oncol 20: 4665-4672, 2002.

10. Johnson DH, Bass D, Einhorn LH, et al: Combination chemotherapy with or without thoracic radiotherapy in limited-stage small-cell lung cancer: a randomized trial of the Southeastern Cancer Study Group. J Clin Oncol 11: 1223-1229, 1993.

11. Osterlind K, Hansen HH, Hansen HS, et al: Chemotherapy versus chemotherapy plus irradiation in limited small cell lung cancer. Results of a controlled trial with 5 years follow-up. Br J Cancer 54: 7-17, 1986.

12. Skarlos DV, Samantas E, Kosmidis P, et al: Randomized comparison of etoposide-cisplatin vs. etoposide-carboplatin and irradiation in small-cell lung cancer. A Hellenic Co-operative Oncology Group study. Ann Oncol 5: 601-607, 1994.

13. Rossi A, Di Maio M, Chiodini P, et al: Carboplatin- or cisplatin-based chemotherapy in first-line treatment of small-cell lung cancer: the COCIS meta-analysis of individual patient data. J Clin Oncol 30: 1692-1698, 2012.

14. Takada M, Fukuoka M, Kawahara M, et al: Phase III study of concurrent versus sequential thoracic radiotherapy in combination with cisplatin and etoposide for limited-stage small-cell lung cancer: results of the Japan Clinical Oncology Group Study 9104. J Clin Oncol 20: 3054-3060, 2002.

15. Skarlos DV, Samantas E, Briassoulis E, et al: Randomized comparison of early versus late hyperfractionated thoracic irradiation concurrently with chemotherapy in limited disease small-cell lung cancer: a randomized phase II study of the Hellenic Cooperative Oncology Group (HeCOG). Ann Oncol 12 1231-1238, 2001

16. Park K, Sun JM, Kim SW, et al: Phase III trial of concurrent thoracic radiotherapy (TRT) with either the first cycle or the third cycle of cisplatin and etoposide chemotherapy to determine the optimal timing of TRT for limited-disease small cell lung cancer. J Clin Oncol 30 (Suppl): abstract 7004, 2012
17. De Ruysscher D, Pijls-Johannesma M, Vansteenkiste J, et al: Systematic review and meta-analysis of randomised, controlled trials of the timing of chest radiotherapy in patients with limited-stage, small-cell lung cancer. Ann Oncol 17: 543-552, 2006.

18. Higgins JPT and Green S (eds): Cochrane Handbook for Systematic Reviews of Interventions Version 5.1.0 [updated March 2011]. The Cochrane Collaboration, 2011.

19. DerSimonian R and Laird N: Meta-analysis in clinical trials. Control Clin Trials 7: 177-188, 1986.

20. Jeremic B, Shibamoto Y, Acimovic L, et al: Initial versus delayed accelerated hyperfractionated radiation therapy and concurrent chemotherapy in limited small-cell lung cancer: a randomized study. J Clin Oncol 15: 893-900, 1997.

21. Work E, Nielsen OS, Bentzen SM, et al: Randomized study of initial versus late chest irradiation combined with chemotherapy in limited-stage small-cell lung cancer. Aarhus Lung Cancer Group. J Clin Oncol 15: 3030-3037, 1997.

22. Spiro SG, James LE, Rudd RM, et al; London Lung Cancer Group: Early compared with late radiotherapy in combined modality treatment for limited disease small-cell lung cancer: a London Lung Cancer Group multicenter randomized clinical trial and meta-analysis. J Clin Oncol 24: 3823-3830, 2006.

23. Murray N, Coy P,Pater JL, et al: Importance of timing for thoracic irradiation in the combined modality treatment of limited-stage small-cell lung cancer. The National Cancer Institute of Canada Clinical Trials Group. J Clin Oncol 11: 336-344, 1993.

24. Perry MC, Herndon JE III, Eaton WL and Green MR: Thoracic radiation therapy added to chemotherapy for small-cell lung cancer: an update of Cancer and Leukemia Group B Study 8083. J Clin Oncol 16: 2466-2467, 1998.

25. Jiang J, Liang X, Zhou X, et al: A meta-analysis of randomized controlled trials comparing irinotecan/platinum with etoposide/platinum in patients with previously untreated extensive-stage small cell lung cancer. J Thorac Oncol 5: $867-873,2010$

26. Hu X, Bao Y, Zhang L, et al: Omitting elective nodal irradiation and irradiating postinduction versus preinduction chemotherapy tumor extent for limited-stage small cell lung cancer: interim analysis of a prospective randomized noninferiority trial. Cancer 118: 278-287, 2012.

27. Gridelli C, Perrone F and Monfardini S: Lung cancer in the elderly. Eur J Cancer 33: 2313-2314, 1997.

28. De Ruysscher D, Pijls-Johannesma M, Bentzen SM, et al: Time between the first day of chemotherapy and the last day of chest radiation is the most important predictor of survival in limited-disease small-cell lung cancer. J Clin Oncol 24: 1057-1063, 2006

29. Pijls-Johannesma M, De Ruysscher D, Vansteenkiste J, et al: Timing of chest radiotherapy in patients with limited stage small cell lung cancer: a systematic review and meta-analysis of randomised controlled trials. Cancer Treat Rev 33: 461-473, 2007.

30. Huncharek M and McGarry R: A meta-analysis of the timing of chest irradiation in the combined modality treatment of limited-stage small cell lung cancer. Oncologist 9: 665-672, 2004. 